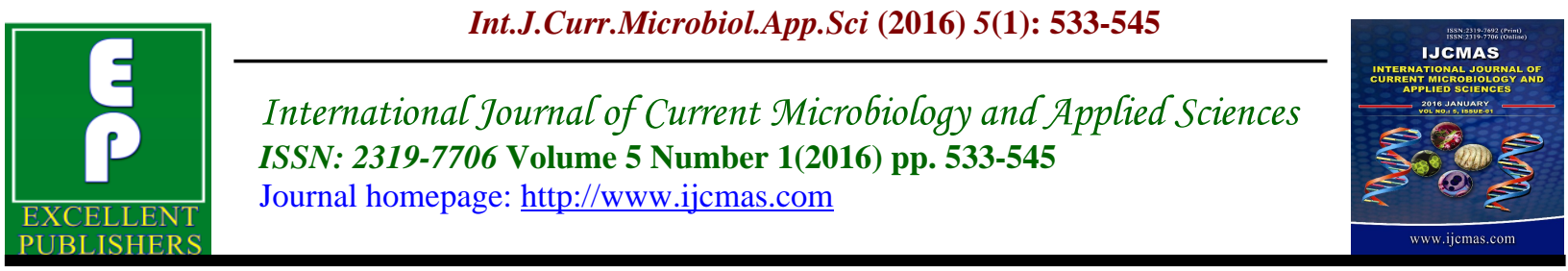

Review Article

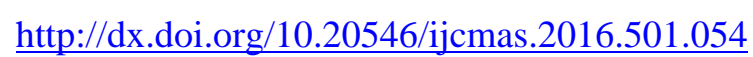

\title{
Why Candida Species have Emerged as Important Nosocomial Pathogens?
}

\author{
Sachin C. Deorukhkar* and Santosh Saini \\ Department of Microbiology, Rural Medical College, Pravara Institute of Medical sciences
}

(Deemed University), Loni, Maharashtra, India

*Corresponding author

\section{A B S T R A C T}

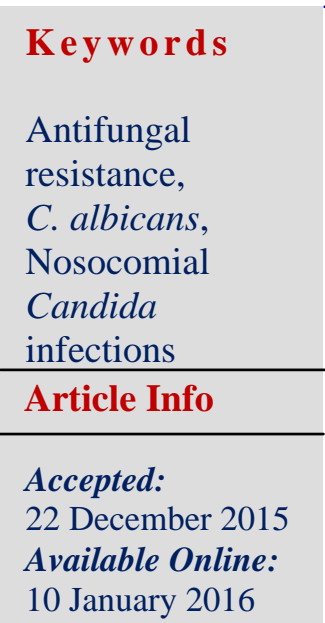

Health care associated infection (HCAI) has a propensity to thwart and decelerate the every effort of clinician taken towards patient care. In recent years HCAIs due to Candida spp. are increasing at an alarming rate. Candida spp. is now considered as the third or fourth most common cause of HCAIs in the United States, out numbering all Gram negative bacilli. Over the past few decades, the species associated with candidiasis has progressively shifted from Candida albicans to treatment resistant non albicans Candida (NAC) spp. Increase in incidence of nosocomial Candida infections and the emergence of unusual, relatively unknown and treatment resistant Candida spp. has emphasized the need of continuous monitoring the spectrum of candidiasis with reference to epidemiology, species distribution and antifungal resistance.

\section{Introduction}

Health care associated infections (HCAIs) or nosocomial infections are infections that occur during hospitalization but are neither present nor incubating upon hospital admission. These infections have occurred since care of patients in hospital began and still continues to haunt health care providers even in the era where medical science has progressed by leaps and bounds.

HCAIs are a major problem in any health care setup, taking its toll in the form of increased mortality and morbidity. They concern $5-15 \%$ of hospitalized patients and can lead to complications in $25-50 \%$ of those admitted in intensive care units (ICUs) (Eggimann and Pittet, 2001).

Any microorganism is capable of causing HCAI, but those that are able to survive and persist in hospital environment for longer period and develop resistance to antimicrobial agents and disinfectants are particularly important.

Advances in medical practice have increased the frequency of fungal infections in hospitalized patients. Fungal pathogens, now account for almost $10 \%$ of all nosocomial blood stream infections (BSIs) (Warnock 
and Campbell, 1996). Among various fungal pathogens, Candida spp. is the important cause of substantial morbidity and mortality in hospitalized patients. It is fast becoming a very important pathogen among critically ill hospitalized patients (Rajkumari et al., 2014).

Candida spp. accounts for $10 \%$ of all bloodstream infections and $25 \%$ of all urinary tract infections (UTIs) in the ICU (Pfaller 1996). Nosocomial candidiasis is associated with prolonged hospitalization and increased health care cost (Lockhart et al., 2014). Candida spp. is now considered as the third or fourth most common cause of HCAIs in the United States, out numbering all Gram negative bacilli (Mean et al., 2008). Over the past few decades, the species associated with candidiasis has progressively shifted from Candida albicans to treatment resistant non albicans Candida (NAC) spp.

Increase in incidence of nosocomial Candida infections and the emergence of unusual, relatively unknown and treatment resistant Candida spp. has emphasized the need of continuous monitoring the spectrum of candidiasis with reference to epidemiology, species distribution and antifungal resistance. Although there are multitudinous meticulously structured literatures available on HCAIs, most of them are focused on bacterial nosocomial pathogens; hence here we present an overview on various factors responsible for emergence of Candida spp. as important nosocomial pathogen.

\section{Methods}

Relevant original and review articles for this review were collected from databases like Pubmed, Scopus and Google Scholar. 'MeSH' terms used for search included 'nosocomial candidiasis' 'candidemia', 'invasive candidiasis and 'fungal infections'.

\section{Candida spp. and its Role in Nosocomial Infection}

Currently there are 200 recognized species of the genus Candida (López-Martínez 2010). The genus Candida includes heterogeneous group of characteristically white and asporogenous yeasts (Deorukhkar and Saini 2015a). Taxonomically, the genus Candida is included in the Phylum Ascomycetes, Class Blastomyces, Order Cryptococcales, Family Cryptococcaceae (Deorukhkar and Saini 2015a). Morphologically Candida spp. are classified as yeast like fungi.

All members of this genus characteristically form true or pseudohyphae (except $C$. glabrata and $C$. parapsilosis. C. glabrata is the only haploid Candida spp. and is incapable of forming both true and pseudohyphae whereas; C. parapsilosis can generate pseudohyphae but not true hyphae) (McCullough et al., 1996; Silva et al., 2012). Candida produces cream to yellow colored colonies on Sabouraud dextrose agar (SDA). Colony texture vary from pasty to dry, smooth to wrinkled and glistening to dry depending on the species (Deorukhkar and Saini 2014).

C. albicans is the most prevalent and pathogenic species responsible for various types of candidiasis. However, over the last few decades the incidence of infections due to NAC spp. has drastically increased.

NAC spp. are emerging as both colonizers and pathogens causing nosocomial fungal BSIs (Krcmery, 2002). C. tropicalis, $C$. glabrata, C. krusei, C. parapsilosis and $C$. lusitaniae are most common isolate from NAC spp. The ARTEMIS Global Antifungal 
Surveillance Program reported $C$. albicans to be the most common cause of candidemia, followed by $C$. glabrata, $C$. tropicalis and C. parapsilosis (Miceli 2011). However, the scenario of species distribution differs according to geographical area and health care set up. $C$. glabrata, the $2^{\text {nd }}$ most common cause of candidemia in USA is less frequently isolated from other countries (Miceli 2011). C. tropicalis and C. parapsilosis are the second and third leading Candida spp. in Brazil whereas $C$. parapsilosis and $C$. glabrata are the most common Candida spp in Australia (Miceli 2011).

C. tropicalis is one of the most common NAC spp. This pathogen alone or in association with other Candida spp. is implicated more frequently in human infections (Deorukhkar et al., 2014a). Studies of various researchers have documented $C$. tropicalis as most common pathogenic species of Candida in cancer patients (Pfaller 1996). In India, $C$. tropicalis is the most common cause of nosocomial candidemia (Giri and Kindo, 2012). C. It is often isolated from ICU patients requiring prolonged catherization (Silva et al., 2012). Jain et al (Jain et al., 2011) reported $C$. tropicalis is the most common cause of candiduria in catheterized ICU patients. Haematogenous infection due to $C$. tropicalis is often associated with high mortality rates (Pfaller 1996). C. tropicalis disseminates at high potency in neutropenic individuals compared with $C$. albicans and other NAC spp (Silva et al., 2012).

C. glabrata, initially regarded as a saprophyte with low virulence has recently emerged as an important nosocomial pathogen (Fidel et al., 1999). It is associated high mortality rates in immunocompromised and at risk hospitalized patients (Deorukhkar and Saini, 2013). Majority of patients with
C. glabrata BSI have rapidly or ultimately fatal disease (Komshian et al., 1989). Prolonged duration of hospitalization and prior antimicrobial use are two major risk factors associated with $C$. glabrata colonization (Fidel et al., 1999). C. glabrata can be acquired directly or indirectly acquired from contaminated environmental surfaces (Fidel et al., 1999). Portal of entry for $C$. glabrata include respiratory and genitourinary tract and surgical wounds (Pfaller 1996). BSI due to C. glabrata is more common among patients with solid tumors and nononcological disorders than among patients with hematologic malignancies (Komshian et al., 1989; Wingard et al., 1995).

C. krusei was considered as a transient commensal of human mucosal surfaces. However, in recent years, it has emerged as an important pathogen causing a wide spectrum of clinical manifestations in hospitalized patients (Samranayake and Samaranayake 1994). It is an important pathogen in granulocytopenic patients (Pfaller 1996). The advent of HIV/AIDS and widespread use of azoles especially fluconazole for prevention and treatment of mycotic infections in this patients have increased the incidence of $C$. krusei infection (Samranayake and Samaranayake 1994).

Although initially considered as nonpathogenic, $C$. parapsilosis has emerged as a significant nosocomial pathogen (Sardi et al., 2013). C. parapsilosis has a high affinity for parenteral nutrition and selectively grow in hyperalimentation solution with high concentration of glucose (Trofa et al., 2008). This emerging notorious pathogen frequently colonizes the hands of healthcare providers and is capable of forming biofilms on prosthetic devices and central venous catheters. It can also persist 
in hospital environment (Clark et al., 2004). In contrast to other Candida spp. disseminated infections due to $C$. parapsilosis can occur without prior colonization and is generally horizontally transmitted via contaminated external sources such as medical devices or fluids, the hands of health care workers, catheters and other implanted devices (Trofa et al., 2008). C. parapsilosis is the predominant Candida spp. isolated in many neonatal intensive care units (NICUs) and is often associated neonatal mortality (Rolides et al., 2004).

C. lusitaniae is an important nosocomial pathogen in immunocompromised hosts. It is most commonly isolated from the respiratory tract, urine and blood. Sanchez et al (1992) reported isolation of C. lusitaniae from hospital environment and subsequent acquisition of the same strain by patients. This Candida spp. is an endogenous pathogen and therefore colonization and infection of a patient is caused by a single strain unique to that individual (Pfaller 1996).

C. kefyr is considered as an emerging pathogen causing nosocomial BSI in neutropenic leukemia patients. Reuter et al (2005) reported C. kefyr BSIs in 3 neutropenic leukemia patients after chemotherapy and/or stem cell transplantation.

C. rugosa is a relatively less common cause of catheter-related Candida BSI. It is has been implicated as a cause of nosocomial BSI in burn and critically ill patients. Less common NAC spp. such as $C$. orthopsilosis, C. metapsilosis and C. nivariensis have been increasingly reported in recent years (Miceli et al., 2011). C. nivariensis can be acquired from hospital gardens or potted plants (Miceli et al., 2011).

\section{Epidemiology of Nosocomial Candidiasis}

Mycotic infections once rarely documented have emerged as important public health problems worldwide. The list of opportunistic fungal pathogens causing serious and fatal infections increases every year (Pfaller and Diekema 2007).The increased incidence of nosocomial fungal infection can be attributed to advances in medical practice, use of broad-spectrum antibiotics and improved laboratory techniques for identification of unusual Candida spp (Ha et al., 2011). Candida is the most important cause of opportunistic mycoses worldwide.

Surgical and ICU patients are at higher risk of nosocomial fungal infections. In ICU patients, the most common types of Candida infections are BSI, catheter-related infections, intra-abdominal infections and UTI (Eggimann et al., 2003). Disseminated Candida infection is a leading cause of morbidity and mortality in both immunocompetent and immunocompromised critically ill patients (Mean $e t$ al., 2008).

In USA, Candida spp. is the fourth leading cause of health care associated BSI and accounts for 8 to $10 \%$ of all nosocomial BSI. It is number three cause of BSI in the ICU and third leading cause of central-lineassociated blood stream infections in the U.S. Candida spp. is the second leading cause of catheter associated UTI in U.S. In European countries disseminated candidiasis accounted for $17 \%$ of nosocomial infections (Eggimann et al., 2003) However, a review of the available literature has revealed a dearth of information on nosocomial candidiasis from developing countries like India. Verma et al (Verma et al., 2003) from Lucknow, India, reported 18- fold increase in candidemia at their hospital in the year 
1995. Prasad et al (Prasad et al., 1999) reported increase in the incidence of nosocomial candidiasis in the year 1997.

Despite antifungal treatment, the mortality rate associated candidemia is high especially in ICU patients (Verduyn Lunel et al., 1999). The average hospital stay is prolonged by 30 days in patients surviving Candida BSI, compared with patients without Candidiasis (Wey et al., 1988).Therefore nosocomial Candida infection is not only life-threatening, but also significantly increase the health care cost.

As Candida spp. is a commensal of gastrointestinal tract, the source of infection for candidiasis usually originate endogenously, however exogenous transmission (patient to patient or healthcare provider to patient) may also occur (Ha 2011). Transmission of Candida spp. to high-risk patients via contaminated infusates, indwelling medical devices, or the hands of health care workers is well documented (Pfaller 1996).

In recent years, a change in the epidemiology of candidiasis from $C$. albicans towards NAC spp. is witnessed by many researchers worldwide. The increase use of azole group of antifungal agents is one of important reason for this shift (Mean et al., 2008). NAC spp. causes $35-65 \%$ of all Candida BSIs in general population (Krcmery and Barnes, 2002). The incidence of BSIs due to NAC spp. is more in cancer patients and bone marrow transplant recipient compared to ICU and surgical patients, children or HIV infected patients (Krcmery and Barnes, 2002). The mortality rate of NAC spp. BSIs is high in ICU and surgical patients compared to cancer patients, children and HIV infected patients (Krcmery and Barnes, 2002). Mortality rates also vary according to the type of Candida spp. C. glabrata and C. krusei infections are usually associated with high mortality rates compared to other azole susceptible NAC spp. (Eggiman et al., 2003).

\section{Risk Factors for Nosocomial Candidiasis}

Like for any other infectious disease, Candida colonization and infection is dependent on host's predisposing factors and pathogenicity of infecting strain. There are multiple risk factors for acquisition of Candida infections. These include surgical intervention, treatment with broad spectrum antibiotics, immunosuppression either induced or acquired, TPN, mechanical ventilation, malignancy, diabetes and prolonged hospitalization. The broader the spectrum and duration of exposure to an antimicrobial agent, the higher the risk of Candida colonization and infection (Eggimann et al., 2003).

Some risk factors like treatment with broad spectrum antibiotics or corticosteroids favors Candida colonization whereas, others like presence of intravascular catheters facilitate dissemination (Ha et al., 2011).

As Candida spp. frequently colonize nonsterile sites in the body (skin, mouth, gut and genitals) (Schelenz 2008), the source infection is usually endogenous. Pittet et al (1994) demonstrated colonization to be an independent risk factor for the development of Candida blood stream infection (Pittet $e t$ al., 1994). Candida colonization increases with the prolonged ICU stay, presence of indwelling urinary catheter and use of antimicrobial agents like vancomycin or imipenem (Charles et al., 2005).

Nosocomial transmission may occur from exogenous sources such as contaminated medical devices and hands of health care 
workers (Ha et al., 2011). Candida spp. can be transmitted from hand to hand and can survive on hands up to $45 \mathrm{~min}$ after inoculation (Rangel-Frausto et al., 1994). Candida can adapt to a variety of different habitats for growth (Seneviratne et al., 2007). It can survive for up to 4 months in the hospital environment (Ha et al., 2011).

Prior treatment or prophylaxis with azole group of antifungal agents is important risk factor for NAC spp. NAC spp. are more frequently isolated from hematology patients with prolonged neutropenia than in nonneutropenic patients in surgical ICU (Ha et al., 2011). Candida colonization, umbilical vessel catheterization, prolonged hospitalization and very low birth weight are important risk factors for nosocomial neonatal candidemia (Narang et al., 1998).

C. krusei infections are more frequent in leukemia patients and bone marrow transplant recipients (Krcmery and Barnes, 2002). Fluconazole prophylaxis is also an important risk factor for $C$. krusei infection (Ha et al., 2011). Risk factors predicting $C$. glabrata infection include the presence of solid tumor, organ transplantation, cardiovascular disease, abdominal surgery, renal disorder and fluconazole prophylaxis (Krcmery and Barnes, 2002). Presence of central venous catheters or other intravascular device and the use of TPN are well documented risk factors for $C$. parapsilosis (Krcmery and Barnes, 2002; Ha et al., 2011). Risk factors for $C$. tropicalis infections include acute leukemia, antineoplastic chemotherapy, neutropenia, ketoconazole prophylaxis and indwelling catheter (Krcmery and Barnes, 2002).

\section{Pathogenicity of Candida spp}

Various virulence factors like adherence to biotic and abiotic substances and production of hydrolytic enzymes play an important role in the pathogenesis of Candida spp (Deorukhkar and Saini 2015 b). Virulence factors interact with host cell and facilitate colonization and progression of infection.

Adherence to host cell is the primary event in Candida colonization and infection (Silva et al., 2012). It is mediated by various non specific factors (hydrophobicity and electrostatic forces) and is progressed by specific adhesins present on the surface of Candida cells. Adhesins recognize ligands such as proteins, fibrinogen and fibronectin on host cells (Li et al., 2003). Candida cells can adhere to variety of host cells including epithelia, endothelia and phagocytic cells. Adherence prevents or at least reduces the extent of yeast clearance by the host defense mechanisms and also ensures the delivery of toxins and enzymes to the host cells.

Nearly half of all cases of nosocomial infections are associated with medical devices (Kojic and Darouiche 2004). Medical device associated infection is the greatest threat against safety in the ICUs. Management of medical device associated infection is difficult and costly affair (Kojic and Darouiche 2004). Candida spp. can form biofilm on most, if not all, medical devices. It can form biofilm on stents, shunts, implants, endotracheal tubes, pacemakers and various types of catheters (Deorukhkar and Saini 2013). Biofilm forming ability is strongly associated with the ability to cause infection and is considered as important virulence determinant during candidiasis (Deorukhkar and Saini 2013). The biofilm production confers certain properties like antifungal drug resistance and protection from host defense mechanism. Biofilm forming Candida isolates are associated with significantly higher mortality rates compared to isolates incapable of forming biofilms (Trofa et al., 2008). 
Production of hydrolytic enzymes is one of the important factors contributing to pathogenesis of Candida. Ability of production of extracellular hydrolytic enzymes varies among the species and also depends on the source or site of infection. Secreted hydrolytic enzymes in Candida spp. include phospholipase, lipase, phosphomonoesterase, hemolysins, hexosaminidase and secreted aspartic proteinase (Sardi et al., 2013). Extracellular hydrolytic enzymes facilitate adherence, tissue penetration, invasion and the destruction of host tissue.

Phospholipases hydrolyze phospholipids into fatty acid and also expose receptors on the host cell membrane to facilitate adherence. Deorukhkar et al (Deorukhkar et al., 2013) reported high phospholipase activity in biofilm producing Candida isolates. Therefore screening of phospholipase activity in biofilm forming Candida spp. can serve as an important parameter to differentiate invasive strains from non invasive colonizers. Ying and Chungyang (Ying and Chunyang, 2011) demonstrated co-relation between high phospholipase activity and resistance to antifungal drugs. Therefore, phospholipase may play an important role in the emergence of azole resistance. The quantity of phospholipase production also varies with the specific isolate and the site of infection. Price et al (Price et al., 1982) reported high phospholipase activity in isolates from BSIs compared to those from wound or urine.

Secreted aspartyl proteinases (Saps) degrade epithelial and mucosal barrier proteins such as collagen, keratin and mucin (Wu et al., 1996). They also degrade complement, cytokines and immunoglobulins (Borst and Fluit, 2003). Saps of pathogenic Candida spp. have been extensively studied. $C$. albicans, C. dubliniensis, C. guilliermondii, C. parapsilosis and $C$. tropicalis possess
SAP gene families (Naglik et al., 2003).

Hemolysins production enables Candida survival and persistence in the host. It degrades hemoglobin and facilitates recovery of the elemental iron from host erythrocytes (Silva et al., 2012). The identification of virulence attributes unique to a particular Candida spp. plays an important role in understanding the mechanism of pathogenesis and epidemiology of candidiasis. In future, these virulence factors may provide powerful insights for development of new antifungal drug targets.

\section{Laboratory Diagnosis}

Nosocomial Candida infections are often severe, rapidly progressive, difficult to diagnose clinically and refractory to therapy (Chakrabarti et al 1999). Therefore rapid and accurate laboratory diagnosis is very important to aid the clinician in timely institution of the appropriate and accurate antifungal treatment. As till today no single diagnostic test provides satisfactory rapid results, the laboratory diagnosis of Candida infections continues to be problematic (Silva et al 2012, Ha et al 2011).

Blood cultures though considered as the "gold standard" technique for diagnosis of disseminated candidiasis lack sensitivity and may require weeks to be positive. New culture media, lysis centrifugation and automated blood culture monitoring systems have decreased the assay time required for isolation of etiological agent. However these techniques have moderate sensitivity. In the study of Berenguer et al (1993) the lysis centrifugation system was found to be positive in only $43 \%$ of autopsy proven cases of systemic Candida infections (Berenguer et al 1993). Automated blood culturing systems like ALERT 3D and BACTEC 9240 have demonstrated 
appreciable sensitivity and specificity for diagnosis of disseminated candidiasis.

Serological techniques can be employed for detection of Candida antigens or antibodies in serum or other body fluids of the patients through immunological or biochemical reactions (Teles and Seixas 2015). These diagnostic techniques are useful for presumptive diagnosis, initiation of antifungal therapy but not for species identification (Teles and Seixas 2015). Galactomannan and (1, 3)- $\beta$-d-glucan are common Candida cell wall antigens targeted for immunodiagnosis of Candida infections. D-arabinitol, enolase and SAP are the examples of other antigens that circulate during Candida infections. Serological assay based on immunoglobulin detection usually lack sensitivity in immunosuppressed due to the low levels of circulating antibodies.

Molecular diagnostic techniques are gaining importance in recent years. These methods are highly accurate, sensitive and specific for identification and differentiation of $C$. albicans from other Candida spp (Neppelenbroek et al 2014). However, many of these methods are expensive and require expertise technical support (Deorukhkar and Saini 2015a).

Molecular diagnostic approach for Candida spp. is either based on polymerase chain reaction (PCR) or non PCR based techniques (Neppelenbroek et al 2014). Various formats of in-house developed and commercial PCR platforms are available for qualitative and/or quantitative in vitro detection of Candida species-specific DNA. These include conventional, semi-nested and nested PCR, PCR-enzyme immunoassay, and different variations of real-time PCR and multiplex PCR .

The Yeast Traffic Light peptide nucleic acid fluorescence in situ hybridization assay (PNA FISH) and Pyrolysis and matrixassisted laser desorption ionization-time of fight mass spectrometry (MALDI-TOF MS) are examples of (Neppelenbroek et al 2014) non PCR based techniques for Candida spp.

\section{Treatment}

Antifungal drugs available for prophylaxis and treatment of disseminated Candida infections differ in their mode of action, pharmacokinetic and pharmacodynamic properties, route of administration, indication, cost and safety (Pfaller et al 2012). Hence it is very essential to consider these factors while selecting an antifungal drug for administration in a particular patient for a particular purpose (Deorukhkar and Saini 2015a).

The purpose for initiation of antifungal treatment in a patient may be prophylaxis, initial (empiric, preemptive or targeted) therapy or salvage therapy (Ruping et al 2008). The antifungal prophylactic therapy is initiated to prevent mycoses in a high-risk population prior to any clinical signs of infections. These include hematopoietic stem cell or solid organ transplant recipients, cancer or critically ill patients, burn patients, immunocompromised hosts or patients undergoing major surgery or treatment involving use of invasive medical devices (Deorukhkar and Saini, 2014). Primary prophylaxis involves the risk of overuse and also promotes the development of antifungal resistance.

Pre-emptive antifungal therapy is administration of an antifungal drug to prevent disseminated Candida infection in a patient with combination of multisite Candida colonization (more than two sites) with at least two major risk factors (prolonged antibiotics, extensive burns, 
immunosuppression, TPN, major abdominal surgery, ileus replacement therapy) or at least three minor risk factors (geriatric age group, length of $>10$ days stay in ICU, invasive catheterization, candiduria, renal insufficiency or diabetes mellitus). It is often started in patients with laboratory results with or without clinical or radiological indications of disseminated fungal infections, but prior to identification of a causative pathogen from sterile site material or tissue specimens.

Empiric antifungal therapy is initiation of antifungal treatment in patients with symptoms suggestive of fungal infection but prior to microbiological or radiological confirmation. The empiric therapy is initiated on the basis of local epidemiology and presenting clinical signs and symptoms (Kontoyiannis 2012). However, the significance of empiric therapy remains controversial, as it may lead to over treatment, unnecessary exposure to toxic drugs and emergence of drug resistant Candida spp. Antifungal drugs available for prophylaxis and treatment of candidiasis belong to 4 principal classes: polyenes, pyrimidines, azoles and echinocandins (Andriole 2000). The polyenes are broadspectrum antifungal drugs. This class of antifungal agents binds to ergosterol, the most important sterol in fungal cell membrane and alters the cell permeability, finally leading to leakage of cellular contents and cell death (Denning and Hope 2010). Amphotericin B (AMB) is the only antifungal polyene that can be administrated systemically. AMB has a broad spectrum antifungal activity against Candida spp. However, some strains of $C$. albicans, $C$. glabrata, C. krusei and $C$. lusitaniae may acquire resistance to $\mathrm{AMB}$ during course of therapy.

Flucytosine (5FC) is an example of pyrimidine analogue used in combination with AMB for life-threatening Candida syndromes like endocarditis, meningitis, and hepatosplenic disease (Dismukes 2000). 5FC affects fungal pyrimidine metabolism by inhibiting RNA and protein synthesis (Andriole 2000).

Azole group of antifungal agents is widely used for prophylaxis and treatment of various types of Candida infections. They block the synthesis of ergosterol (major component of fungal cytoplasmic membrane) by inhibiting p450 dependent enzyme sterol 14- $\alpha$-demethylase. The inhibition of this enzyme results in increased membrane permeability and ceasation of cell growth and reproduction (Ha et al 2011, Kathiravan et al 2012).

The azole class has two subclasses the imidazoles (ketoconazole, micoconazole and clotrimazole) and the triazole (fluconazole, itraconazole, voriconazole and posaconazole). The presence of a third nitrogen atom in the core structure distinguishes triazoles from imidazoles. Fluconazole is extensively used for prophylaxis and treatment of superficial and disseminated candidiasis (Kathiravan et al 2012). Many recent studies have suggested the contribution of empirical fluconazole prophylaxis in shift towards NAC spp.

Echinocandins are new addition to the antifungal armamentarium against Candida spp. These group of antifungal agents target the cell wall and inhibit 1,3- and 1,6,- $\beta$-Dglucan synthesis. Caspofungin, micafungin and anidulafungin are examples of echinocandins approved by FDA. All three compounds of echinocandins have fungicidal action (both in vitro and in vivo) against most Candida spp irrespective of their resistance or susceptibility to azole or amphotericin B (Denning 2003; Giri and Kindo 2012). In recent years, use of echinocandins has significantly increased in 
many hospitals. Echinocandins (especially caspofungin) are now considered as firstline drugs for treatment of disseminated candidiasis among patients who are critically ill, clinically unstable, or have a recent exposure or colonization with a Candida spp. known to have reduced susceptibility to azole (Kauffman et al 2006).

\section{Conclusion}

Health care associated infections (HCAIs) are increasing at an alarming rate. It leads to increased mortality, morbidity and consequently extended hospital stay and expenses. Hence, HCAIs need to be curbed through awareness, a change in our practice and constant vigilance. In recent years HCAIs due to Candida spp. are increasing at an alarming rate. Over the past few decades the trend of Candida infections has away from $C$. albicans to treatment resistant non albicans Candida spp. This review was structured to encompass the key factors responsible for emergence of Candida spp. as an important nosocomial pathogen.

\section{Acknowledgement}

We are grateful to the management of Rural Medical College and Rural Hospital of Pravara Institute of Medical Sciences, Deemed University, Loni, Maharashtra, India for their encouragement and support throughout the study.

\section{Conflict of Interest}

The authors declare that there no conflict of interests regarding the publication of this paper.

\section{References}

Andriole, V. 2000. Current and future antifungal therapy: new targets for antifungal therapy. International $J$. Antimicrob. Agents. 16:317-21.

Berenguer, J., Buck, M., Witebsky, F., Stock, F., Pizzo, P., and Walsh, T. 1993, Lysis-centrifugation blood cultures in the diagnosis of tissueproven invasive candidiasis: disseminated versus single-organ infection. Diagn. Microbiol. Infect. Dis. 17:103-109.

Borst, A., and Fluit, A. 2003, High levels of hydrolytiv enzymes secreted by Candida albicans isolates involved in respiratory infections. J. Med. Microbiol. 52: 971-74.

Chakrabarti, A., Singh, K., Das, S. 1999, Changing face of nosocomial candidemia. Ind. J. Med. Microbiol. 17: 160-166.

Charles, P., Dalle, F., Aube, H., et al., 2005, Candida spp. colonization significance in critically ill medical patients: a prospective study. Intensive Care Med. 31:393-400.

Clark, T., Slavinski, S., Morgan, T., et al. 2004, Epidemiologic and molecular characterization of an outbreak of Candida parapsilosis blood stream infections in a community hospital. $J$. Clin.Microbiol. 42:4468-72.

Deorukhkar, S., and Saini, S. 2013, Evaluation of phospholipase activity in biofilm forming Candida species isolated from intensive care unit patients. British Microbiol. Res. J. 3: 440-7.

Deorukhkar, S., and Saini, S. 2014, Virulence markers and antifungal susceptibility profile of Candida glabrata: An emerging pathogen. British Microbiol. Res. J. 4: 35-45.

Deorukhkar, S., and Saini, S. 2014, Laboratory approach for diagnosis of candidiasis through ages. Int. J. Curr. Microbiol. App. Sci. 3: 206-218. 
Deorukhkar, S., Saini, S., and Mathew, S. 2014 a, Virulence factors contributing to pathogenicity of Candida tropicalis and its antifungal susceptibility profile. Int. J. Microbiol., vol. 2014, Article ID 456878, 6 pages.

Deorukhkar, S., Saini, S., and Mathew, S. 2014 b, Non- albicans Candida infection: An emerging threat. Interdiscip Perspect Infect Dis, vol. 2014, Article ID 615958, 7 pages, 2014.

Deorukhkar, S., and Saini, S. 2015a, Candidiasis: Past, present and future. Int. J. Infect. Trop. Dis. 2:12-24.

Deorukhkar, S., and Saini, S. 2015b, Virulence factors attributed to pathogenicity of non albicans Candida species isolated from human immunodeficiency virus infected patients with oropharyngeal candidiasis. Annals of Pathology and Laboratory Medicine. 3: A62-5.

Denning, D., and Hope,W. 2010, Therapy for fungal diseases: opportunities and priorities. Trends Microbiol. 18:195204.

Dismukes, W. 2000. Introduction to antifungal drugs. 30: 653-7.

Eggimann, P., and Pittet, D. 2001, Infection control in the ICU. Chest. 120: 205993.

Eggimann, P., Garbina, J., Pittet, D. 2003, Epidemiology of Candida species infections in critically ill nonimmunosuppressed patients. Lancet Infect. Dis. 3:685-702.

Giri, S., and Kindo, A. 2014, A review of Candida species causing blood stream infection. Indian J. Med. Microbiol. 30: 270-8.

Ha, J., Italiano C., Heath, C., Shih, S., Rea, S., and Wood, F. 2011, Candidemia and invasive candidiasis: A review of literature for the burns surgeon. Burns. 9:181-95.
Jain, M., Dogra, V., Mishra, B., Thakur, A., Loomba, S., and A. Bhargava. 2011, Candiduria in catheterized intensive care unit patients: emerging microbiological trends. Indian J. Pathol. Microbiol. 54:552-555.

Kathiravan, M., Salake, A., Chothe, A., et $a l ., 2012$, The biology and chemistry of antifungal agents: A review. Bioorg. Med. Chem. 20: 5678-98.

Kojic, E., Darouiche, R. 2004, Candida infections of medical devices. Clin. Microbiol. Rev. 17:255-67.

Komshian, S., Uwaydah, A., Sobel. J., and Crane, L. 1989, Fungemia caused by Candida species and Torulopsis glabrata in hospitalized patient: frequency, characteristics, and evaluation of factors influencing outcome. Rev. Infect. Dis. 11:379-90.

Kontoyiannis, D. 2012, Invasive mycoses: strategies for effective management. Am. J. Med. 125:S25-38.

Krcmery, V., and Barnes, A. 2002, Nonalbicans Candida spp. causing fungaemia: pathogenicity and antifungal resistance. J. Hosp. Infect. 50: 243-260.

Li, X., Yan, Z., Xu, J. 2003, Quantitative variation of biofilms among strains in natural populations of Candida albicans. Microbiol. 149:353-62.

Lockhart, S. 2014, Current epidemiology of Candida infection. Clin. Microbiol. Newsl. 36: 131-36.

McCullough, M., Ross, B., and Reade, P. 1996, Candida albicans: a review of its history, taxonomy, epidemiology, virulence attributes, and methods of strain differentiation. Int. J. Oral Maxillofac. Surg. 25: 136-44.

Méan, M., Marchetti, O., and Calandra, T. 2008, Bench-to-bedside review: Candida infections in the intensive care unit. Crit. Care.12: article 204. 
Miceli, M., Diaz, J., and Lee, S. 2011, Emerging opportunistic yeast infections. Lancet Infect. Dis. 11:14251.

Naglik, J., Challacombe, S., and Bernhard, H. 2003, Candida albicans secreted aspartyl proteinases in virulence and pathogenesis. Microbiol. Mol. Bio. Rev.67: 400-28.

Narang, A., Agarwal, P., Chakrabarti, A., and Kumar, P. 1998, Epidemiology of systemic candidiasis in a tertiary neonatal unit. J. Trop. Paed. 44:104-8.

Nepplenbroek, K., Seo, R, Urban, V., et al., 2014, Identification of Candida species in the clinical laboratory: a review of conventional, commercial, and molecular techniques. Oral Dis. 20: 329-44.

Pfaller, M. 1996, Nosocomial candidiasis: Emerging species, reservoirs, and modes of transmission. Clin. Infect. Dis. 22: S89-94.

Pittet, D., Monod, M., Suter, P., Frenk, E., Auckenthaler, R. 1994, Candida colonization and subsequent infections in critically ill surgical patients. Ann. Surg. 220: 751-8.

Prasad, K., Agarwal, J., Dixit, A., Tiwari, D., Dhole, T., and Ayyagari, A. Role of yeasts as nosocomial pathogens and their susceptibility to fluconazole and amphotericin B. Indian J. Med. Res. 110:11-7.

Price, M., Wilkinson, I., Gentry, L. 1982. Plate method for detection of phospholipase activity in Candida albicans. Sabouraudia. 20:7-14.

Pfaller, M. and Diekema, D. 2007, Epidemiology of invasive candidiasis: A Persistent public health problem. Clin. Microbiol. Rev. 20:133-63.

Rajkumari, N., Mathur, P., Xess, I., and Misra, M. 2014, Distribution of different yeasts isolates among trauma patients and comparison of accuracy in identification of yeasts by automated method versus conventional methods for better use in low resource countries. Indian J. Med. Microbiol. 32: 391-94.

Rangel-Frausto, M., Houston, A., Bale, M., $\mathrm{Fu}$, C., and Wenzel, R. 1994, An experimental model for study of Candida survival and transmission in human volunteers. Eur. J. Clin. Microbiol. Infect. Dis. 13:590-5.

Reuter, C., Morgan, M., Bange, F., et al. 2005, Candida kefyr as an emerging pathogen causing nosocomial bloodstream infections in neutropenic leukemia patients. Clin. Infect. Dis. 41:1365-6.

Roilides, E., Farmaki, J., Evdoridou, J., et al. 2004, Neonatal candidiasis: analysis of epidemiology, drug susceptibility, and molecular typing of causative isolates. Eur. J. Clin. Microbiol. Infect. Dis. 23:745-50.

Samaranayake, Y., and Samaranayake, L.1994, Candida krusei: biology, epidemiology, pathogenicity and clinical manifestation of an emerging pathogen. J. Med. Microbiol. 41:295310.

Sanchez, V., Vazquez, J., Barth-Jones, D., Dembry, L., Sobel, J., Zervos, M. 1992, Epidemiology of nosocomial acquisition of Candida lusitaniae. $J$. Clin. Microbiol. 30:3005-8.

Sardi, J., Scorzoni, L., Bernardi, T., FuscoAlmedia, A., Mendes Giannini, M. 2013, Candida species: current epidemiology, pathogenicity, biofilm formation, natural antifungal products and new therapeutic options. J. Med. Microbiol. 62:10-24.

Schelenz, S. 2008, Management of candidiasis in intensive care unit. J Antimicrob. Chemother. 61:i31-i34.

Seneviratne, L., Jin, L., Samaranayake, L. 2008, Biofilm lifestyle of Candida: a minireview. Oral Dis. 14:582-90. 
Silva, S., Negri, M., Henriques, M., Oliveria, R., Williams, D., and Azeredo, J. 2012, Candida glabrata, Candida parapsilosis and Candida tropicalis: biology, epidemiology, pathogenicity and antifungal resistance. FEMS Microbiol. Rev. 36:288-305.

Teles, F., and Seixas, J. 2015, The future of novel diagnostics in medical mycology. J. Med. Microbiol. 64:315-22.

Trofa, D., Gácser, A., and Nosanchuk, J. 2008, Candida parapsilosis, an emerging fungal pathogen. Clin. Microbiol. Rev. 21:606-25.

Verduynlunel, F., Meis, J., and Voss, A. 1999, Nosocomial fungal infections: candidemia. Diagn. Microbio.l Infect. Dis. 34: 213-20.

Verma, A., Prasad, K., Singh, M., Dixit, A., and Ayyagari, A. 2003, Candidaemia in patients of a tertiary health care hospital from north India. Indian $J$. Med. Res. 117:122-8.
Warnock, D., and Cambell, C. 1996, Medical Mycology. Mycol Res. 100: 1153-62.

Wey, SB., Mori, M., Pfaller, MA.,Woolson, RF., and Wenzel, RP. 1988. Hospitalacquired candidemia. The attributable mortality and excess length of stay. Arch Intern Med. 148:2642-2645.

Wingard, J.1995, Importance of Candida species other than $C$. albicans as pathogens in oncology patients. Clin. Infect. Dis. 20:115-25.

Wu, T., Samaranayake, L., Cao, B., and Wang, L. 1996, In-vitro proteinase production by oral Candida albicans isolates from individuals with and without HIV infection and its attenuation by antimycotic agents. $J$ Med Microbiol. 44:311-16.

Ying, S., and Chunyang, L., Correlation between phospholipase of Candida albicans and resistance to fluconazole. Mycoses. 55:50-55.

\section{How to cite this article:}

Sachin C. Deorukhkar and Santosh Saini. 2016. Why Candida Species have Emerged as Important Nosocomial Pathogens? Int.J.Curr.Microbiol.App.Sci. 5(1): 533-545 ihttp://dx.doi.org/10.20546/ijcmas.2016.501.054 